\title{
GaAs, InP, InGaAs, GaInP, p+-i-n+ Multiplication measurements for Modeling of Semiconductor as photo detectors
}

\author{
Sanjay.C.Patil \\ (Research Scholar at NMIMS MUMBAI) \\ Parshvanath College of Engineering, THANE (W), \\ Mumbai, 400601 India
}

\author{
B.K.Mishra \\ Thakur College of Engineering and Technology, \\ Kandivali (E) Mumbai, 400101 India
}

\begin{abstract}
Optoelectronic is one of the thrust areas for the recent research activity. One of the key components of the optoelectronic family is photo detector to be widely used in broadband communication, optical computing, optical transformer, optical control etc. Present paper includes the investigation. carried on the basis of the. Multiplication measurements on GaAs, InP, InGaAs, GaInP, p+-i-n+s with region thicknesses, with investigation of applicability of the local ionization theory. A local ionization coefficient to be increasingly unrepresentative of the position dependent values in the device as is reduced below $1 \mathrm{um}$.
\end{abstract}

Keywords-Photo detectors; Impact ionization.

\section{INTRODUCTION}

With the advent of broadband communication, INTERNET communication, optical computing, the research in the optical devices has emerged as on of the thrust area for the active and consistent research. One of such primary devices is the photo detector. Therefore, recent years have seen tremendous research activities in the area of photo detector mathematical modeling and simulation. It includes the domain like optical control of r.f./microwave devices, broadband communication like optical communication, as a optoelectronic component to support green technology, etc.

Basic objective of the active research includes the reduction in the device dimension, reduction in power utilization, better performance against noise, ease of integration to make complete system on chip, better reliability against all possible adverse condition and so on. At present the commercially available photo detectors for the various includes PIN diode, APD, photo transistor and the recent device like PIN FET.A photo detector operates by converting light signals that hit the junction to a voltage or current. The junction uses an illumination window with an anti-reflect coating to absorb the light photons. The result of the absorption of photons is the creation of electron-hole pairs in the depletion region. Impact Ionization is the process in a material by which one energetic charge carrier can lose energy by the creation of other charge carriers. For example, in semiconductors, an electron (or hole) with enough kinetic energy can knock a bound electron out of its bound state (in the valence band) and promote it to a state in the conduction band, creating an electron-hole pair Three-five materials refer to compound semiconductors made from one element from
Group III on the periodic chart (arsenic in the case of GaAs) and one from Group V (gallium in the case of GaAs). Other three-five (or III-V in Roman numerals) semiconductors include indium phosphide and gallium nitride.

The band gap energy of semiconductors tends to decrease with increasing temperature. When temperature increases, the amplitude of atomic vibrations increase, leading too larger inters atomic spacing. In a regular semiconductor crystal, the band gap is fixed owing to continuous energy states. In a quantum dot crystal, the band gap is size dependent and can be altered to produce a range of energies between the valence band and conduction band. It is also known as quantum confinement effect.

Band gaps also depend on pressure. Band gaps can be either direct or indirect, depending on the electronic band structure.

\section{Gallium arsenide (GaAs)}

A compound of the elements gallium and arsenic it is an III/V semiconductor, and is used in the manufacture of devices such as microwave frequency integrated circuits, monolithic microwave integrated circuits, infrared light-emitting diodes, laser diodes, solar cells, and optical windows.

\section{Indium phosphide (InP)}

A binary semiconductor composed of indium and phosphorus. It has a face-centered cubic crystal structure, identical to that of GaAs and most of the III-V semiconductors $\mathrm{InP}$ is used in high-power and high frequency electronics because of its superior electron velocity with respect to the more common semiconductors silicon and gallium arsenide. It also has a direct bandgap, making it useful for optoelectronics devices like laser diodes.

\section{Indium gallium arsenide (InGaAs)}

Indium gallium arsenide (InGaAs) is a semiconductor composed of indium, gallium and arsenic. It is used in highpower and high-frequency electronics because of its superior electron velocity with respect to the more common semiconductors silicon and gallium arsenide. InGaAs band gap also makes it the detector material of choice in optical fiber communication at 1300 and $1550 \mathrm{~nm}$. Gallium indium arsenide (GaInAs) is an alternative name for $\mathrm{InGaAs}$.

\section{Gallium indium Phosphide (GaInP)}


GaInP, a wide band gap semiconductor lattice matched to $\mathrm{GaAs}$, is of interest for a variety of device applications such as heterojunction bipolar transistors (HBTs) And heterojunction field-effect transistors (HFETs) The advantages of GaInP over GaAlAs for GaAs-based HBT applications include its large energy band gap $(1.9 \mathrm{eV})$, lower conduction band offset, reduced deep-level concentration, and easier selective etching. GaInP-emitter HBTs with high current gain and good microwave performance have been reported

\section{THEORY}

To design structures for high-voltage or high-power applications, an accurate knowledge of impact ionization coefficients in GaInP is necessary for calculating breakdown characteristics of junctions. In the work reported here, photocurrent multiplication was used to measure the electron and hole ionization coefficients in $\sim 100 \mathrm{GaInP}$ by illuminating $p^{+}-n^{-}-n^{+}$diode structures from either side with above band gap radiation.6 The results show that GaInP has significantly lower values of $a$ and $b$ than those of GaAs or InP, a promising indication for high-voltage applications.

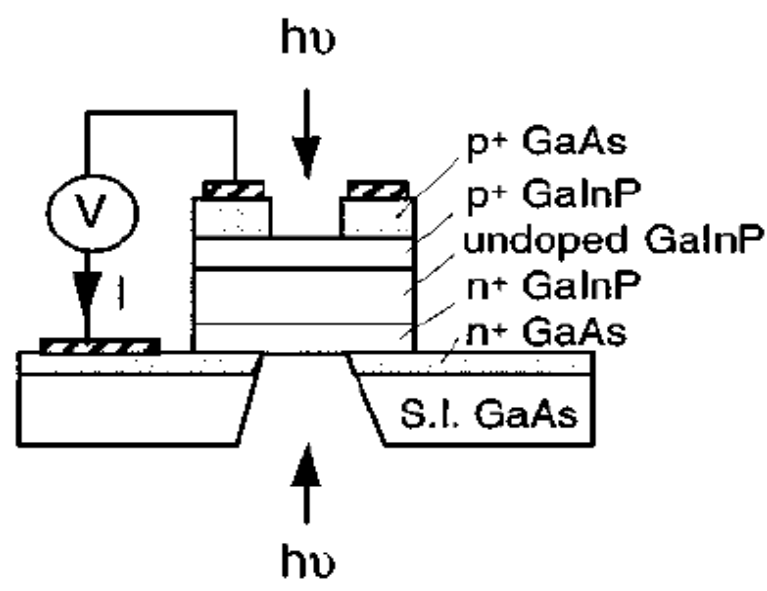

Fig.1. Basic structure of photo detector

Accurate determination of the electron and hole ionization coefficients, $\alpha$ and $\beta$ respectively, is important, since these are used to determine avalanche multiplication characteristics and breakdown. Conventionally $\alpha$ and $\beta$ are assumed to depend only on the local electric field, and the mean multiplication due to an electron-hole pair generated at position is given by

$$
M\left(x_{0}\right)=\frac{\exp \left[-\int_{x_{0}}^{W}(\alpha-\beta) d x\right]}{1-\int_{0}^{W} \alpha \exp \left[-\int_{0}^{x}(\alpha-\beta) d x\right] d x}
$$

As described by Stillman and Wolffe [7] where $\mathrm{W}$ is the total depletion width. The electric field exists between $\mathrm{x}=0$ and $\mathrm{x}=\mathrm{W}$ causing electrons to move from left to right. This expression is also traditionally used to derive the values of, $\alpha$ and $\beta$ from photo multiplication measurements performed with carrier injection from the depletion region edges. [7]-[8]

For electron multiplication, $M_{e} \quad x_{o}=0$ and for pure hole multiplication $M_{h}, \quad x_{o}=\mathrm{W}$. However, carriers entering the high field region with energy much less than the ionization threshold must traverse a dead space, distance $d_{e}$, for electrons or $d_{h}$ for holes, before they acquire sufficient energy to impact ionize. With estimation this dead space distance is given by equating it to the ballistic distance a carrier requires to reach the ionization threshold energy,

$$
E_{t h} \text { i.e. } d=E_{t h} / q F \text {. }
$$

For a device where electrons are injected at $x=0$, these corrections usually disallow electron ionization in a deadspace region from $0<x<d_{e}$ and hole ionization in the region from $W-d_{h}<x<W$. Okuto and Crowell [9] presented an approximate expression relating multiplication to the ionization coefficients while accounting for the reduced multiplication by these regions. Bulmanet al[10]. simplified their expression to interpret the measured multiplication results from $\mathrm{p}-\mathrm{n}-\mathrm{n}$ junctions by assuming no electron-initiated ionization occurs within a distance $d_{e}$ from their injection point.

\section{Physics of Impact Ionization}

Avalanche photo detectors (APDs) use the impact ionization principle to detect and amplify very low light signals and they are still used as the most sensitive detector for most systems. It is found that to have a high-gain, low-noise APD, the ionization rate for electrons and holes needs to be very different. Efforts to find the best material to perform impact ionization reveal that silicon has the largest ionization rate difference between electrons and holes among all semiconductors. Due to its cut-off wavelength around $1 \mu \mathrm{m}$, however, silicon has rarely been employed for today's optical communication systems that use $1.55 \mu \mathrm{m}$ wavelengths light. Suitable materials using combinations of binary and ternary/quaternary III-V semiconductors such as InGaAs on InP substrates have provided solutions for longer wavelength detection.

\section{Impact Ionization Gain Mechanism}

The impact ionization gain mechanism can be demonstrated by using a thermally generated electron, or an electron created by an absorbed photon, travelling inside a semiconductor where a depletion region is formed. Figure 6 illustrates the impact ionization process in a reverse biased PIN. As shown in the figure, electrons can gain sufficient kinetic energy while travelling in a high electric field. If the electric field is high enough, this high-energy electron may initiate the electron-electron scattering so that an electron in the valence band can be excited to the conduction band. As a result of this, another electron-hole pair is produced by promoting an electron from the valence band into the conduction band. Due to the strong electric field, the subsequent electron and hole will continue to collide with the 
lattice and create more electron-hole pairs. Therefore, numerous carriers are generated and the result is a multiplied current output. This phenomenon is sometimes referred to as the avalanche breakdown

\section{Ionization Threshold Energy}

The minimum energy required to excite an electron, however, is more than just freeing an electron from the valence band. In order to decide the ionization threshold energy, various methods including from the valence to the conduction band is equal to the band gap energy of the semiconductor. Impact ionization parabolic, non parabolic, realistic, and non local pseudo potential band structure have been utilized. It is found that the threshold energy differs for most semiconductors. It is a function of the band structure, effective mass ratio between electron and hole, density of state, phonon interaction, and spin-orbit splitting energy.

The best way to estimate the ionization threshold energy involves a simple two parabolic band model. In this model, we consider one conduction band with effective mass $m_{e}$ and one valence band with mass $m_{h}$. As shown in Fig. 6, prior to the collision, the electron travelling from the left-hand side has a kinetic energy of $1 / 2 m_{e} v_{i}^{2}$ and a momentum of $m_{e} v_{i}$, where $v_{i}$ is the initial velocity of the electron. After collision, three carriers exist: a new electron-hole pair plus the original electron. Electrons continue travelling to the right and the hole to the left.

Assuming that the collision is elastic, the conservation of energy and momentum must be satisfied these two assumptions are summarized as

$$
\frac{1}{2} m_{e} v_{t}^{2}=E_{g}+\left(\frac{1}{2} m_{e} v_{e}^{2} \times 2+\frac{1}{2} \times m_{h} v_{h}^{2}\right)
$$

And

$m_{e} v_{t}=m_{e} v_{i} \times m_{h} v_{h}$

Where $E_{g}$ is the energy band gap of the semiconductor, $v_{e}$ is the electron velocity, and $v_{h}$ is the hole velocity after the collision. Note that when $m_{e}=m_{h}$ and $v_{e}=v_{h}=v_{i}$, (1) and (2) can be derive that the required initial electron energy for ionization process as

$$
E_{i}=\frac{1}{2} m_{e} v_{i}^{2}=1.5 E_{g}
$$

This is the well-known 3/2-band-gap rule for ionization threshold energy in semiconductor [1].

\section{Ionization Coefficients and Gain Equations}

To derive the gain equations for impact ionization process, we need to first define the impact ionization coefficient. For most semiconductor materials, the impact ionization process is asymmetric to some degree for electrons and holes. For example, the ionization rate for electrons is about 5 times greater than holes in Germanium. For silicon, however, electrons can ionize 50 times to 1000 times more readily than holes depending on the electric field amplitude. The probability for initiating impact ionization is quantified as the impact ionization coefficient. It is defined as the reciprocal of the average distance travelled by an electron or hole to produce an electron-hole pair. Therefore, its unit is $\mathrm{cm}^{-1}$. For electrons, the coefficient is denoted as $\alpha$. For holes, it is denoted as $\beta$. Materials with very different value for $\alpha$ and $\beta$, such as in silicon can create less impact ionization noise because only one type of carrier is dominant during the ionization process. First, the length of the semiconductor is long $(\mathrm{L}>1 \mu \mathrm{m})$ such that non-local theories [4] are not considered. Secondly, the current density is low to avoid the space charge effect which may screen the electric field and lower the gain. A differential equation can be used to describe the multiplication process in terms of ionization rates $\alpha$ and $\beta$. If $\beta$ is much smaller than $\alpha$, (4) can be rewritten as

$$
M_{n}=\frac{1}{1+\frac{\alpha}{\alpha-\beta}[\exp (-x(\alpha-\beta))]_{0}^{L}}=\frac{(\alpha-\beta) \exp (L(\alpha-\beta))}{\alpha-\beta \exp (L(\alpha-\beta))}
$$

$M_{p}=\frac{1}{1+\frac{\beta}{\beta-\alpha}[\exp (-x(\beta-\alpha))]_{0}^{L}}=\frac{(\beta-\alpha) \exp (L(\beta-\alpha))}{\beta-\alpha \exp (L(\beta-\alpha))}$

$M_{n} \approx \frac{\exp (L \alpha)}{1-\frac{\beta}{\alpha} \exp (L \alpha)}$

A positive feedback factor $(\beta / \alpha)$ in (6) shows the effect of ionization coefficients on multiplication gain. As $\beta$ gets bigger, but still much smaller than $\alpha, M_{n}$ can reaches breakdown in a shorter distance.

If we consider an extreme case, $\beta=0,(2.6)$ can be further simplified to

$M_{n}=\exp \left(\int_{0}^{L} \alpha d x\right)=\exp (\alpha L)$

From (7) we observe that when $\beta=0$, there is no avalanche breakdown because $M_{n}$ just continues to increase exponentially with $\alpha L$. A plot is given in Fig.2 using (6) to demonstrate the gain versus $\alpha L$ for various value of $(\alpha / \beta)$

\section{Impact Ionization Coefficient Measurement}

We know that $\alpha$ and $\beta$ are strong function of electric field in the multiplication region. Electric field, however, also depends on the bias voltage, doping profile, and the device geometry. In this section, we will explain how to measure the ionization coefficients accurately for different device structures including p-i-n diodes. 
In measurement, a very high energy light is used to illuminate the diode. Due to the high energy of the photons, electron-hole pairs can be created very close to the surface to obtain the pure electron or hole injection condition. Electron ionization is measured by shining light from the $\mathrm{p}+$ side. Photon-excited electrons are injected into the intrinsic layer while holes are swept to the left. Hole ionization is measured by illuminating light from the $n+$ side, injecting holes into the intrinsic layer.

The electron gain $M_{n}$ can be calculated by dividing the total current measured for a given electric field by the photocurrent created by the light. The photocurrent can be precisely measured when the diode is operated without avalanche gain. The hole gain $M_{p}$ can be obtained through the same process

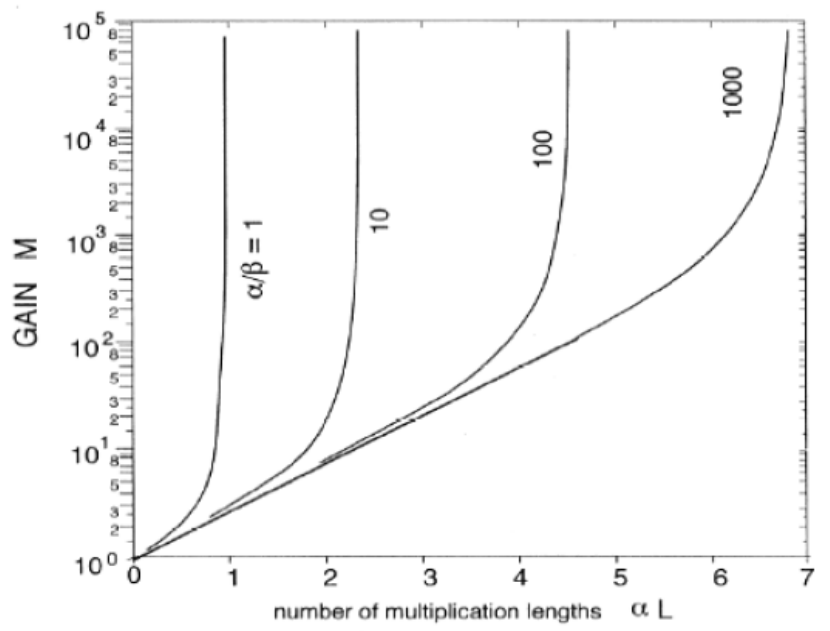

Fig.2. Multiplication gain $\mathbf{M}$ versus $\mathbf{\alpha} \mathbf{L}$ for pure electron injection. Various $\alpha / \beta$ value are used to demonstrate its effect on avalanche breakdown curve

. Once $M_{n}$ and $M_{p}$ are known, we can use equation (4) and (5) to derive formulas related $\alpha$ and $\beta$ to $M_{n}$ and $M_{p}$. They are calculated to be

$\alpha=\frac{1}{L}\left(\frac{M_{n}-1}{M_{n}-M_{p}}\right) \ln \left(\frac{M_{n}}{M_{p}}\right)$

And

$$
\beta=\frac{1}{L}\left(\frac{M_{p}-1}{M_{p}-M_{n}}\right) \ln \left(\frac{M_{p}}{M_{n}}\right)
$$

\section{Impact Ionization Response}

The carrier build-up time in the multiplication process depends on the contribution of carrier feedback In the ideal condition, where only one type of carrier is capable of initiating impact ionization process (electrons for $\beta=0$ ), the output current pulse increases with the transit time for the initially injected electron. The output current decreases to zero as all the ionized holes arrive at the negatively biased electrode. Thus, the current pulse lasts about twice as long as the transit time. Since the pulse width is independent of the multiplication gain, there is no gain-bandwidth product limitation when $\beta$ or $\alpha=0$.

It is found that the avalanche multiplication process does not affect the device bandwidth as long as the dc multiplication gain $\mathrm{M}$ is less than $\alpha / \beta$. On the other hand, if $\mathrm{M}$ $>\alpha / \beta$, the multiplication gain becomes a function of frequency and is expressed as

$$
M(\omega)=M_{0} /\left(\omega^{2} M_{0}^{2} \tau_{1}^{2}\right)^{1 / 2}
$$

Where $\tau 1$ is an effective transit time and is approximately $\tau 1=\mathrm{N}(\beta / \alpha) \tau$. $\mathrm{N}$ is a number varying slowly from $1 / 3$ to 2 as $\beta / \alpha$ varying from 1 to $10^{-3}$, and $\tau$ is the transit time equal to $L / v_{s}$ where $\mathrm{L}$ is the length of the avalanche gain region and $v_{s}$ is the saturation velocity

The gain-bandwidth product for $M>\alpha / \beta$ can be obtained using (10) for high frequencies and is expressed as

$$
M(\omega) \times \omega=\frac{M_{0} \omega}{\left(\omega^{2} M_{0} \tau_{1}^{2}\right)^{1 / 2}}=\frac{1}{\tau_{1}}=\frac{1}{N(\beta / \alpha) \tau}
$$

Equation (11) indicates the basic requirements for an impact-ionization based device to obtain a high gainbandwidth product. These requirements include a small $\beta / \alpha$ value and short intrinsic time. Therefore, a correct choice of material, multiplication layer thickness, and carrier transport velocity are essential.

Using (10), the calculated frequency response for a P-I-N structure with $1 \mu \mathrm{m}$ thick gain region when operating at $\mathrm{M}=50$ is given in Figure 3.

Using Matlab to simulate the both the measure of $\alpha$ and $\beta$ since Bulmanet al [10]. found that $\beta>\alpha$, these two sets of parameters for the coefficients since they enable the data to be more accurately quantified over the wide field range the coefficients are parameterized in The impact ionization coefficients can be fitted into an exponential form:

$$
\begin{aligned}
& \alpha(E)=A_{e} \exp \left(-B_{e} / E\right) \\
& \beta(E)=A_{h} \exp \left(-B_{h} / E\right) \\
& \alpha_{e H}=A_{e H} \exp \left[-\left(B_{e h} / \xi\right)^{C_{e H}}\right. \\
& \beta_{h}=A \exp \left[-(B / \xi)^{C}\right.
\end{aligned}
$$

For the local calculation the values of $\alpha$ and $\beta$ were taken from.[10] for electric fields, F, This investigation involved measuring both the multiplication and excess noise 
characteristics of several samples with overlapping field regions and represents the most extensive and rigorous to date

Impact Ionisation coefficient and Multiplication of electrons \& holes for GaAs

Impact Ionisation Coefficients for GaAs

$$
\begin{aligned}
& \alpha(E)=1.89 \times 10^{7}\left[\left(\exp \left(-\left(5.75 \times 10^{7}\right) / E\right)^{1.82}\right] \mathrm{cm}^{-1}\right. \\
& \beta(E)=2.21 \times 10^{7}\left[\left(\exp \left(-\left(6.57 \times 10^{7}\right) / E\right)^{1.75}\right] \mathrm{cm}^{-1}\right.
\end{aligned}
$$

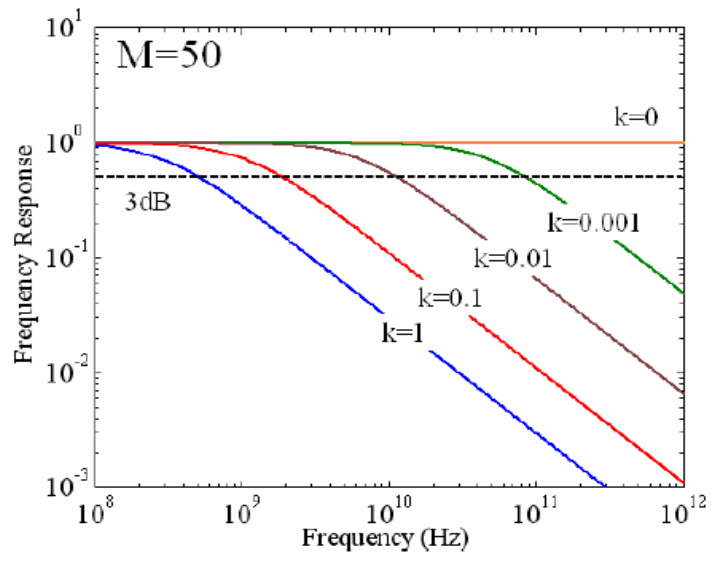

Fig.3. Frequency Response of a P-I-N photodiode with $1 \mu \mathrm{m}$ thick multiplication layer. Notice that parameter $\mathrm{k}$ is define as $\beta / \alpha$

\section{Multiplication of electrons and holes for GaAs}

Simulation of calculate Electron and hole multiplication factors for a range of ideal $\mathrm{p}$-i-n $\mathrm{s}$ with from $1 \mathrm{um}$ down to 0.48 um for GaAs with temperatures of $20 \mathrm{~K}$ to $500 \mathrm{~K}$, Shown in figure 5 increasing temperature causes the multiplication of electrons and holes to shift to higher voltage therefore higher electric fields are required to offset the increase in carrier cooling by phonon scattering and maintain multiplication

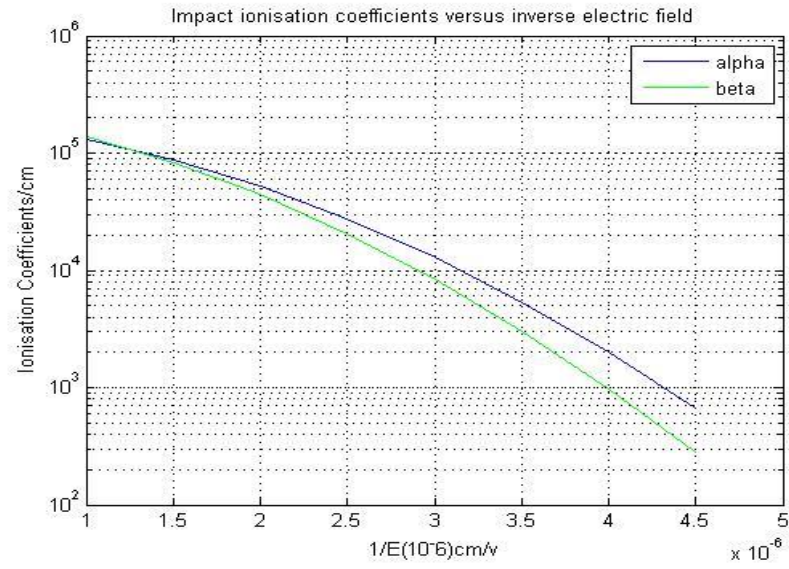

Fig.4. Impact Ionisation Coefficients for GaAs versus inverses electric field

Impact Ionisation coefficient and Multiplication of electrons \& holes for InGaAs $\alpha_{e H}=A_{e H} \exp \left[-\left(B_{e h} / \xi\right)^{C_{e H}}\right.$

$\beta_{h}=A \exp \left[-(B / \xi)^{C}\right.$

Impact Ionisation Coefficients for InGaAs (narrowband gap semiconductor) great importance in the case of an ionization coefficient which increases with temperature that can result in an unstable positive power dissipation feedback which the voltage breakdown can be obtained by extrapolating the multiplication curveAs shown in fig 6

For alpha

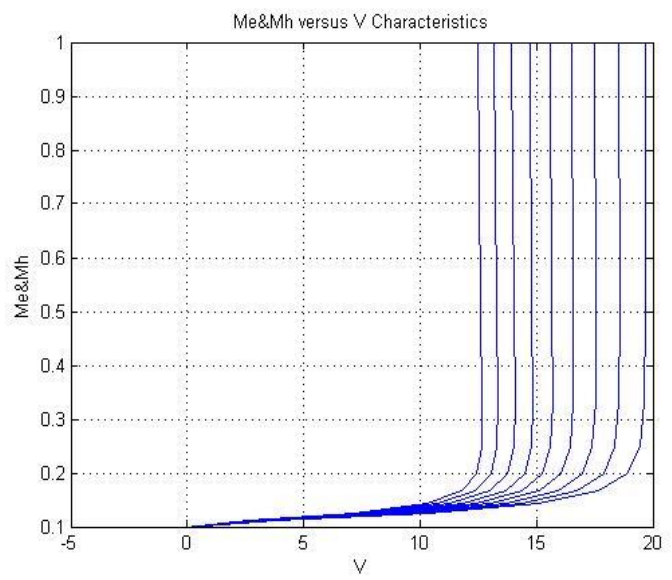

Fig.5. simulated of multiplication $w=0.48 \mu m$ device as a function of temperature from $20 \mathrm{~K}$ to $500 \mathrm{~K}$

$$
\begin{aligned}
& A_{e H}=7.2597 \times 10^{4}-(24.204 T)+\left(0.3259 T^{2}\right) \mathrm{cm}^{-1} \\
& B_{e H}=5.9988 \times 10^{5}+\left(3.4763 \times 10^{2} T\right)+\left(2.4768 T^{2}\right) \mathrm{V} / \mathrm{cm} \\
& C_{e H}=1.783+\left(7.2548 \times 10^{-4} \mathrm{~T}\right)
\end{aligned}
$$

For beta

$$
\begin{aligned}
& A=6.1026 \times 10^{5}+\left(9.6637 \times 10^{2} T\right)+\left(1.1384 T^{2}\right) \mathrm{cm}^{-1} \\
& B=1.3394 \times 10^{6}+\left(1.0699 \times 10^{3} T\right)+\left(20.4507 T^{2}\right) \mathrm{V} / \mathrm{cm} \\
& C=1.0910-\left(2.3505 \times 10^{-4} T\right)
\end{aligned}
$$

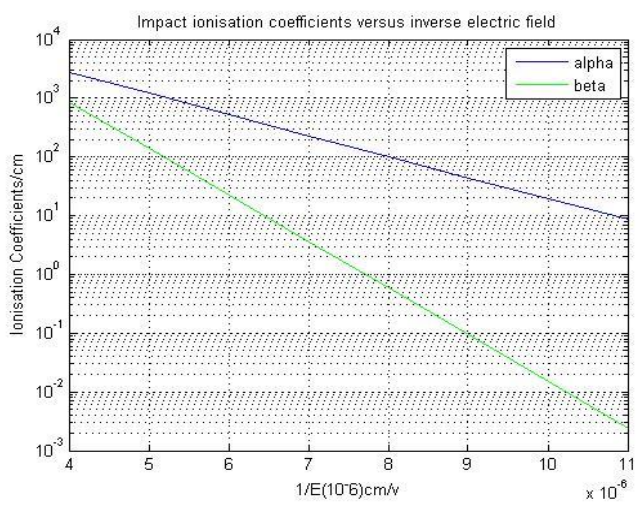

Fig.6. Impact Ionisation Coefficients for InGaAs versus inverses electric field

Multiplication of electrons and holes for InGaAs 
Simulation of Electron multiplication factors that depends on temperature with the thickness of $1.3 \mathrm{um}$ and $1.9 \mathrm{um}$ for InGAas p-i-ns from $20 \mathrm{~K}$ to $400 \mathrm{~K}$ and hole multiplication with thickness of 3.0um as n-i-p from 20K-300K.

The results from figure 7 show a very limited increase in photocurrent initially and then the sudden and clear onset of the avalanche multiplication process. $\mathrm{T}$

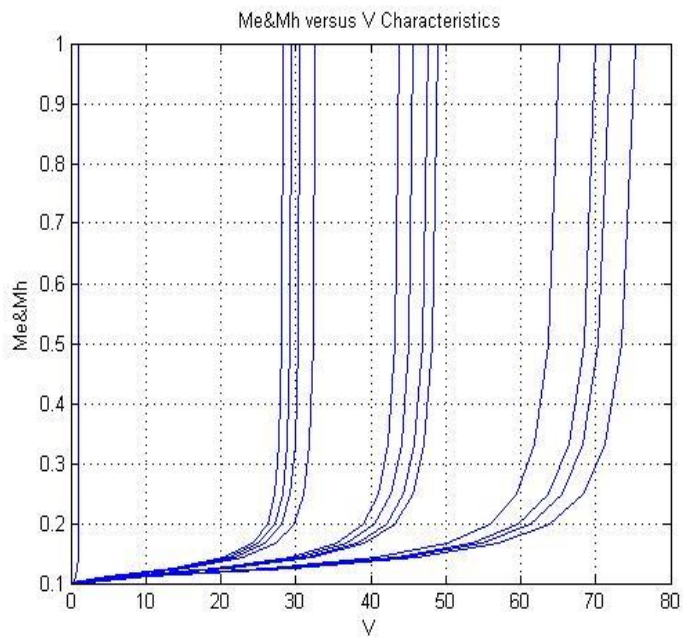

Fig.7. Simulated of multiplication characteristics of 1.3 and 1.9 um thick InGaAs p-i-n diode from $20-400 \mathrm{~K}$

\section{Impact Ionisation coefficient and Multiplication of electrons \& holes for InP}

The electron and hole ionization coefficients can be extracted from the measured multiplication results if both electrons initiated and hole initiated multiplication results are available for the same structure When an electron (or hole) initiates themultiplication process, an electric current is induced by the moving electronsand holes within the multiplication region

InP has high electron peak velocities resulting from large inter valley separation and good breakdown properties owed to a relatively low electron impact ionization coefficient

Compared to the data of GaAs and InP illustrated in Fig.6 and Fig. 8, GaInP has the lowest values of $\alpha$ and $\beta$, signifying a higher breakdown voltage. Moreover, the slopes of the curves of $\alpha$ and $\beta$ vs $1 / E$ for GaInP shown in Fig. 7 are steeper than for the others.

Impact Ionisation Coefficients for InP

$\alpha(E)=2.93 \times 10^{6}\left[\exp \left(-\left(2.64 \times 10^{6}\right) / E\right] \mathrm{cm}^{-1}\right.$
$\beta(E)=1.62 \times 10^{6}\left[\exp \left(-\left(2.11 \times 10^{6}\right) / E\right] \mathrm{cm}^{-1}\right.$

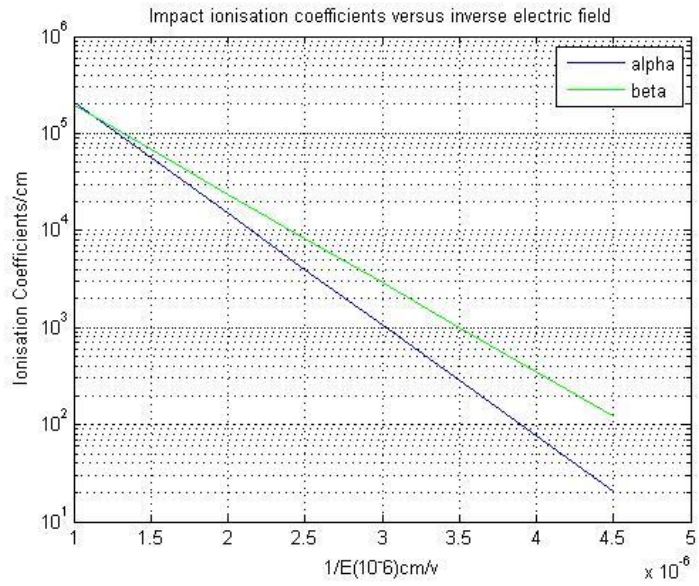

Fig.8. Impact Ionisation Coefficients for InP versus inverses electric field

\section{Multiplication of electrons and holes for InP}

The results from figure 9 show that the temperature dependence of multiplication is not uniform, since the multiplication curves with thickness of the materials from 0.24 to 2.40um respectively. Simulation of Electron multiplication factors for a range of ideal $\mathrm{p}$-i-ns with from 0.24um down to $2.40 \mathrm{um}$ for $\mathrm{InP}$ with temperatures of $20 \mathrm{~K}$ to $500 \mathrm{~K}$

As a test of the parameterized coefficients, test shows for all the materials, the predicted multiplication for an ideal diode, compared with the measured characteristic. It can be seen that the agreement is good, both at low and high multiplications using matlab for these simulations for wideband gap semiconductor and narrowband gap semiconductor.

The structures for high-voltage or high-power applications, an accurate knowledge of impact ionization coefficients in GaInP is necessary for calculating breakdown characteristics of junctions.

In the work reported here, photocurrent multiplication was used to measure the electron and hole ionization coefficients from either side with above bandgap radiation.

The results show that GaInP has significantly lower values of $\alpha$ and $\beta$ than those of GaAs or InP, a promising indication for high-voltage applications. 


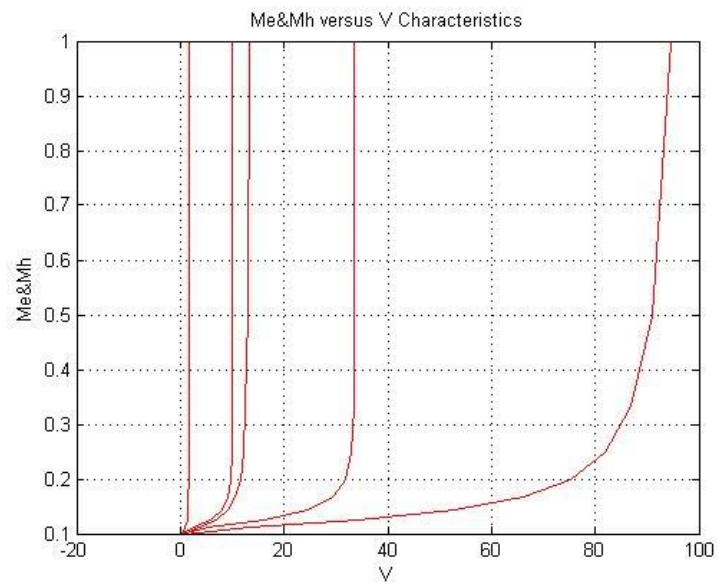

Fig.9. Simulated of multiplication characteristics of 0.24 and 2.40 um thick for InP p-i-n diode from $20-400 \mathrm{~K}$

Impact Ionisation coefficient and Multiplication of electrons \& holes for GaInP

Impact Ionisation Coefficients for $\mathrm{GaInP}$ (wideband gap semiconductor)

$$
\begin{aligned}
& \alpha(E)=3.85 \times 10^{6}\left[\exp \left(-\left(3.17 \times 10^{6}\right) / E\right] \mathrm{cm}^{-1}\right. \\
& \beta(E)=1.71 \times 10^{6}\left[\exp \left(-\left(3.19 \times 10^{6}\right) / E\right] \mathrm{cm}^{-1}\right.
\end{aligned}
$$

The electron and hole multiplication factors, $\mathrm{Mn}$ and $\mathrm{Mp}$, are defined as the total output photocurrent divided by the electron or hole current injected at the contacts. Results for $\mathrm{Mn}$ and $\mathrm{Mp}$ from respective measurements are shown in Fig 10 .

In these results, slight corrections were made to the injected currents due to slightly voltage-dependent intrinsic reverse currents. Because the widths of the depletion regions depend on bias, the number of thermally generated minority carriers that diffuse into the depleted $n^{-}$region also varies. This leads to a slight increase of the injected current as the reverse bias is increased

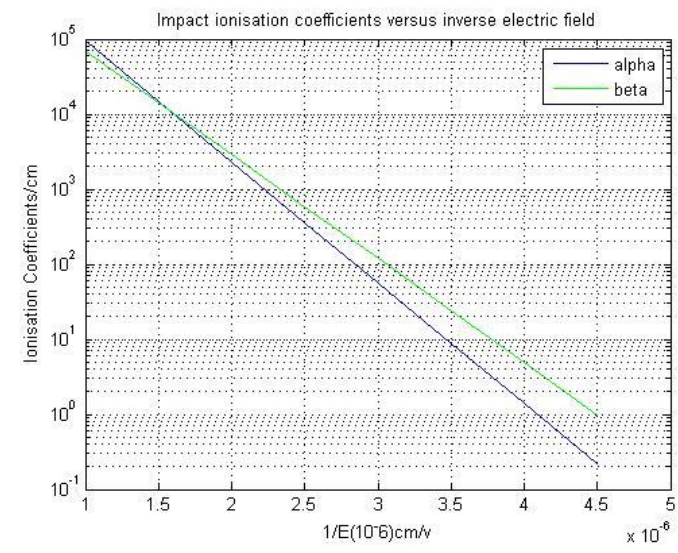

Fig.10. Impact Ionisation Coefficients for GaInP versus inverses electric field

Multiplication of electrons and holes for GAInP
Simulation of Electron multiplication factors for a range of ideal $\mathrm{p}$-i-n $\mathrm{s}$ with from $0.24 \mathrm{um}$ down to 2.40um for GaInP with temperatures of $300 \mathrm{~K}$

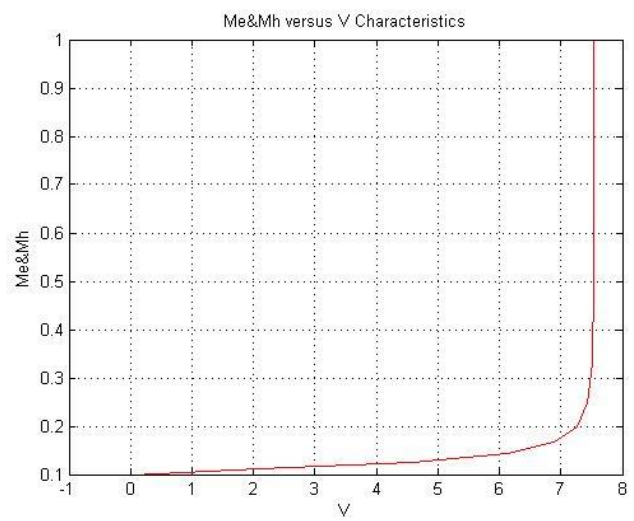

Fig.11. Simulated of multiplication characteristics of 0.24 um thick for GaInP p-i-n diode from $300 \mathrm{~K}$

\section{RESULT AND DISCUSSION}

Voltage breakdown due to avalanche multiplication, also defined as the reverse bias voltage where multiplication rate goes to infinity naturally of great practical interest.

The voltage $\mathrm{VB}$ is defined as the reverse-bias voltage across the multiplication region at which the mean gain becomes infinite.

In doing so rather then testing with hands on materials in fab or clean room physically testing the materials, with this simulation we can create a model and simulate any materials first with respect to their voltage breakdown versus thickness (width) without wasting unnecessary time and cost for testing of materials

\section{Case 1}

GaAs wide band gap, highly resistive which makes it a very good electrical substrate therefore makes it a very good material for ideal material for microwave and millimeter wave integrated circuits. It can also be operated at higher power levels because they have higher breakdown voltage.

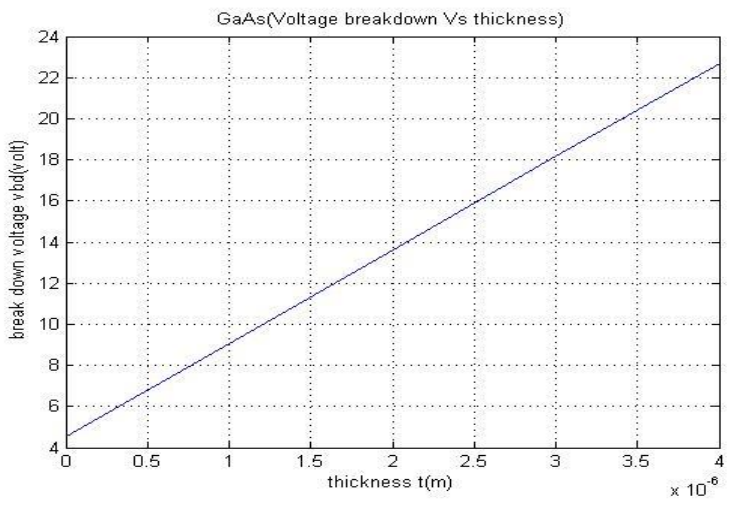


Fig.12. Simulated of voltage breakdown versus thickness for GaAs

\section{Case 2}

GaInP wideband gap semiconductor exhibits high breakdown voltage characteristics indicating that it is a good choice of material for high power applications. This suggests that the ideal electric field distribution assumption is valid and that edge effects are not important in this material system for mesa geometry structures. Note that since these punches through devices have a low carrier concentration in the $n^{-}$ region and high breakdown voltage, the effects of dead space could be ignored. $\alpha$ And $\beta$ data were used to calculate the expected breakdown voltages for $\mathrm{p}-\mathrm{i}-\mathrm{n}$ diode with various thicknesses

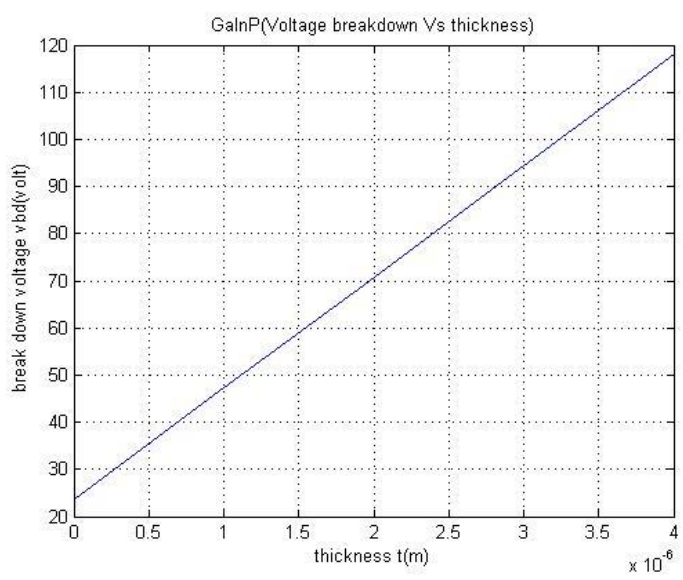

Fig.12. Simulated of voltage breakdown versus thickness for GaInP

GaInP wideband gap semiconductor exhibits high breakdown voltage characteristics indicating that it is a good choice of material for high power applications. This suggests that the ideal electric field distribution assumption is valid and that edge effects are not important in this material system for mesa geometry structures. Note that since these punches through devices have a low carrier concentration in the $n^{-}$ region and high breakdown voltage, the effects of dead space could be ignored. $\alpha$ And $\beta$ data were used to calculate the expected breakdown voltages for $\mathrm{p}-\mathrm{i}-\mathrm{n}$ diode with various thicknesses

\section{Case 3}

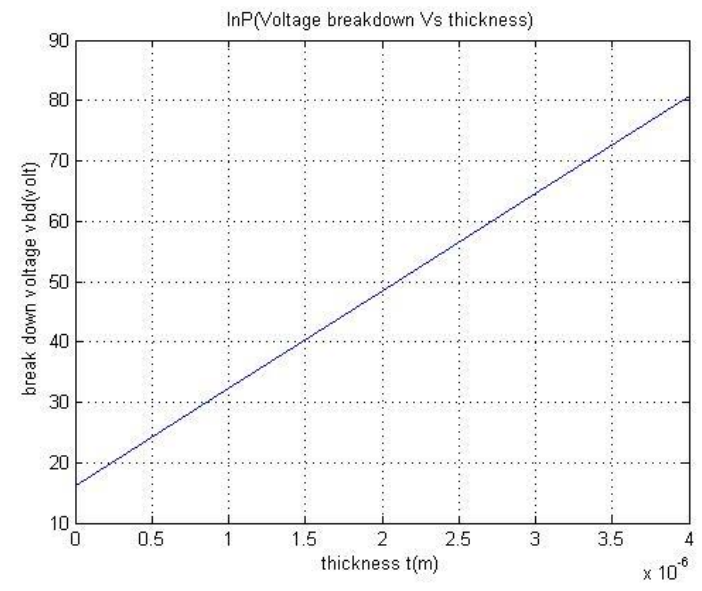

Fig.13. Simulated of voltage breakdown versus thickness for InP

InP wideband gap have an advantage compared to GaAs for many applications when used in high-field regions of the device profile, can significantly improve device performance. These applications include high performance power amplifier for cellular phones, ultra-efficient ultra-linear power amplifiers ideally suited for digital communication systems and satellite networks ICs, and highly integrated mixed signal and high-speed fiber-optic circuits. Wide bandgap semiconductors are associated with a high breakdown voltage. This is due to a larger electric field required to generate carriers through impact mechanism.

\section{Case 4}

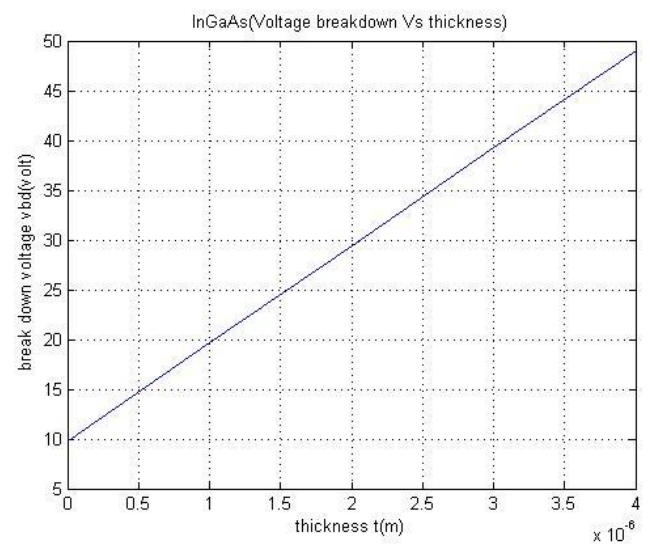

Fig.14. Simulated of voltage breakdown versus thickness for InGaAs

InGaAs with narrow band gap is also a popular material in infrared detectors and some short wave infrared cameras. It also has lower multiplication noise than germanium when used as the active multiplication layer of an avalanche photodiode. Also has a low breakdown voltage

\section{CONCLUSION}

For this research work materials are simulated base on $\mathrm{p}$-i$\mathrm{n}$ diodes therefore very little studies on Avalanche Photodiode (APD) and Schottky Barrier diodes.

The carriers are absorbed in a $\pi$ - region. The absorption leads to the generation of electron-hole pairs in this region. 
The electric field in the $\pi$-region is high enough to separate the carriers. The electric field across the $\pi$-region is not high enough for the charge carriers to gain enough energy for multiplication to take place.

\section{REFERENCES}

[1] [1] B.K.Mishra,computerAidedmodelingof solidstate photodetectors Ph.DthesisBirlainstituteoftechnology,Mesra,Ranchi 1995

[2] T. P. Pearsall, "Impact ionization rates for electrons and holes in Ga0.47In 0.53As,” Appl. Phys. Lett. 36, 218-220 (1980)

[3] S. Wang, R. Sidhu, X. G. Zheng, X. Li, X. Sun, A. L. Holmes, Jr., and J. C. Campbell, IEEE Photonics Technol. Lett. 13, 1346 (2001).

[4] B.K.Mishra, LochanJolly, S.C.Patil," $\operatorname{In}_{1-x} \mathrm{Ga}_{x} A$ s a next generation material for photodetectors ",JSAM.2011

[5] R.Poerschke,Shpringer-Verlag,Madelung,O.(ed.),Semiconductor: group IV elements and III-V compound. Series "Data in science and technology, Berlin, 164 (1991).

[6] J. R. Chelikowsky, and M. L. Cohen, Phys. Rev. B14, 556 (1976).

[7] R. J. McIntyre, "A New Look at Impact Ionization - Part I: A Theory of Gain, Noise, Breakdown Probability, and Frequency Response," IEEE Trans. Electron Devices, vol. 46, 1623-1631 (1999).

[8] H. Ando and H. Kanbe, "Ionization coefficient measurement in GaAs by using multiplication noise characteristics," Solid-State Electron., vol. 24pp. 629-634, 1981.

[9] O.Konstantinov, Q.Wahab, N. Nordell, and U. Lindefelt, "Ionization rates and critical fields in 4H silicon carbide," Appl. Phys. Lett., vol. 71, July 1997

[10] Y.Okuto and C. R. Crowell, "Ionization coefficients in semiconductors," Phys. Rev. B., vol. 10, pp. 4284-4296, Nov. 1973.

[11] G. E. Bulman, V. M. Robbins, and G. E. Stillman, "The determination of impact ionization coefficients in (100) gallium aresenide using avalanche noise and photocurrent multiplication measurements," IEEE Trans. Electron Devices, vol. ED-32, pp. 2454-2466, Nov. 1985.
[12] M. M. Hayat, B. E. A. Saleh, and M. C. Teich, "Effect of dead space on gain and noise of double-carrier-multiplication avalanche photodiodes," IEEE Trans. Electron Devices, vol. 39, pp. 546-552, Mar. 1992.

[13] M. M. Hayat, W. L. Sargeant, and B. E. A. Saleh, "Effect of dead space on gain and noise in Si and GaAs avalanche photodiodes," IEEE J. Quantum Electron, vol. 28, pp. 1360-1365, May 1992.

[14] A. Di Carlo and P. Lugli, "Dead-space effects under near breakdown conditions in $\mathrm{AlGaAs} / \mathrm{GaAs} \mathrm{HBT}$ 's," IEEE Electron Device Lett., vol. 14, pp. 103-105, Mar. 1993.

[15] S. P. Wilson, S. Brand, and R. A. Abram, "Avalanche multiplication properties of GaAs calculated from spatially transient ionization coefficients," Solid-State Electron., vol. 38, pp. 2095-2100, Nov. 1995.

[16] A. Di Carlo and P. Lugli, "Dead-space effects under near breakdown conditions in $\mathrm{AlGaAs} / \mathrm{GaAs}$ HBT's," IEEE Electron Device Lett., vol. 14, pp. 103-105, Mar. 1993.

[17] S. P. Wilson, S. Brand, and R. A. Abram, "Avalanche multiplication properties of GaAs calculated from spatially transient ionization coefficients," Solid-State Electron., vol. 38, pp. 2095-2100, Nov. 1995.

[18] IEEE TRANSACTIONS ON ELECTRON DEVICES, VOL. 50, NO. 10, OCTOBER 20032027

[19] IEEE TRANSACTIONS ON ELECTRON DEVICES, VOL. 50, NO. 10, OCTOBER 20032021 Temperature Dependence of Breakdown and Avalanche Multiplication in In0:53Ga0:47As Diodes and Heterojunction Bipolar Transistors M. Yee, W. K. Ng, J.P. R. David, Senior Member, IEEE, P. A. Houston, C. H. Tan, and A. Krysa

[20] Shanghai Institute of Technical Physics Theory Study of SAGCM InP L. Lin, W. J. Wang, N. Li, X. S. Chen and W. Lu Shanghai Institute of Technical Physics, Chinese Academy of Sciences National Lab for Infrared Physics,

[21] Impact ionization coefficients in (100) GaInP S.-L. Fu, T. P. Chin, M. C. Ho, C. W. Tu, and P. M. Asbeck Department of Electrical and Computer Engineering, University of California, San Diego, La Jolla, California 92093 